\title{
Catalytic oxidation of methane over PdO in wire microcalorimetry
}

\author{
Taichang Zhang ${ }^{\text {a,d,e }}$, Yuxuan Xin ${ }^{\mathrm{a}}$, Zhuyin Ren ${ }^{\mathrm{b}}$, Fei Qi ${ }^{\mathrm{d}}$, Chung K. Law ${ }^{\mathrm{a}, \mathrm{c}, *}$ \\ a Department of Mechanical and Aerospace Engineering, Princeton University, Princeton, NJ 08544, USA \\ ${ }^{\mathrm{b}}$ Department of Mechanical Engineering, University of Connecticut, Storrs, CT 06269, USA \\ c Center for Combustion Energy, Tsinghua University, Beijing 100084, China \\ ${ }^{\mathrm{d}}$ National Synchrotron Radiation Laboratory, University of Science and Technology of China, Hefei, Anhui 230029, China \\ e State Key Laboratory of High Temperature Gas Dynamics, Institute of Mechanics, Chinese Academy of Sciences, Beijing 100190, China
}

\section{A R T I C L E I N F O}

\section{Article history:}

Received 2 May 2012

Received in revised form 3 September 2012

Accepted 7 September 2012

Available online 3 October 2012

\section{Keywords:}

Catalytic oxidation

Pd-based catalyst

Methane combustion

Mechanism

\begin{abstract}
A B S T R A C T
A two-dimensional model of a weakly buoyant flow over a horizontal wire with surface reaction was developed, using a literature mechanism, to simulate the heat release rate of the catalytic oxidation of methane ( 2 vol.\% in air) over a Pd wire with a porous $1-2 \mu \mathrm{m}$ PdO surface layer, acquired by wire microcalorimetry over the temperature range of $600-770 \mathrm{~K}$. The experimental and simulation results demonstrate that the catalytic oxidation is characterized by a low-temperature, reaction-controlled regime in which the internal pore surface is totally accessible to the reactions, and a high-temperature regime in which finite-rate pore diffusion also affects the overall heat release rate. Furthermore, the controlling reactions are identified to be the oxidative adsorption of methane, desorption of oxygen, and adsorption of oxygen, with the former two being facilitating and the last retarding. The reaction mechanism was modified using the response surface methodology and the experimental data in the low-temperature reaction-controlled regime, yielding satisfactory prediction of the global activation energy and identification of the role of oxygen coverage in the transition of the global activation energy.
\end{abstract}

(C) 2012 The Combustion Institute. Published by Elsevier Inc. All rights reserved.

\section{Introduction}

The potential use of methane as a fuel for aero-propulsion is based on the consideration of its greater heat sink capacity, reduced coking propensity than conventional jet fuels, and its higher volumetric energy density than hydrogen in the cryogenic state. Methane, however, is difficult to ignite, and as such presents a significant handicap for such severely residence-time-limited application as supersonic propulsion. Recently it has been suggested that ignition can be catalytically facilitated by seeding the flow with palladium (Pd) or palladium oxide (PdO) nanoparticles generated in situ from a soluble Pd precursor [1].

Since Pd-based catalysts have higher reactivity for methane than other catalysts, the oxidation of methane over them has been extensively studied [2-12]. These studies were mostly focused on certain global aspects of the catalytic process, such as the adsorption of methane [3-5] and oxygen [9], water poisoning [6-8], and the oxidation of Pd [12]. However, only few surface mechanisms were reported. In particular, Sidwell and co-workers proposed a 15-reaction surface mechanism to describe their stagnation flow

\footnotetext{
* Corresponding author. Department of Mechanical and Aerospace Engineering, Princeton University, Princeton, NJ 08544, USA

E-mail address: cklaw@princeton.edu (C.K. Law).
}

experimental data in the catalyst temperature range of 673$1033 \mathrm{~K}[10]$, and deduced a global surface mechanism in the temperature range of 953-1383 K [11]. Furthermore, Shimizu et al. [1] experimentally studied the catalytic ignition of methane over in situ generated Pd-based nano-catalyst in a flow tube. A surface mechanism was developed to simulate the experimental data, with good agreement. Experiments were also conducted [13] on the catalytic ignition of methane over PdO using wire microcalorimetry, which offers a well-defined geometry, flow field and catalyst surface. The catalytic ignition temperature and heat release rate were measured for various methane/air concentrations and system pressures, from which the global activation energy and reaction order were extracted.

In view of the simplicity in concept and design of wire microcalorimetry, yielding a well-defined two-dimensional weaklybuoyant flow field which is amenable to accurate computational simulation, the purpose of the present investigation is to simulate the experiment of [13], and through it acquire further understanding of the catalytic ignition process. Our interest includes the assessment and improvement of the catalytic reaction mechanism compiled in [1], the influence of the surface morphology of the catalytic wire on the extent of the catalytic heat release rate, as well as the cause for the existence of a transition temperature in the global activation energy. 


\section{Experimental specifications}

We briefly describe the experimentation in order to define the experimental situation adopted in the simulation. Wire microcalorimetry [13-15] involves placing a straight catalytic wire in a steady, low-speed gas flow in the cross-stream orientation. By passing an electric current through it, the wire is heated and its temperature is determined through a balance between Joule heat generation and heat loss due to radiation, conduction, and convection to the gas stream. Thus by passing a certain current $(I)$ through the wire, and also by measuring its resistance $(R)$, the power required to maintain the wire at temperature $T$ as $P=I^{2} R$ can be evaluated, with $T$ simultaneously determined by the property relation $R(T)$. Since there is catalytic heat release at the wire surface, less current is needed to maintain the wire at a certain temperature in the reactive flow, consisting of a mixture of air and fuel, as compared to the nonreactive flow of air. The difference in the power inputs determined in the nonreactive and reactive environments is the catalytic heat release rate $(\Delta P)$ of the surface reaction. Experiments for different temperatures were conducted up to $780 \mathrm{~K}$ as the wire temperature becomes non-uniform for higher temperatures, caused by the end effect of thermal conduction.

The Pd wire used in the experiment was $105 \mathrm{~mm}$ in length and $0.1 \mathrm{~mm}$ in diameter (99.99\%, Aldrich). Methane (99.98\%, Airgas) and high purity air $\left(21 \% \mathrm{O}_{2}+79 \% \mathrm{~N}_{2}\right.$, Airgas) were premixed to obtain 2 vol.\% methane in air. The wire was subjected to a welldefined pre-treatment process [13] leading to the formation of a porous surface layer of PdO. Surface analysis [13] demonstrated conclusively that, after pre-treatment and throughout experimentation, a 1-2 $\mu \mathrm{m}$ thick PdO layer is formed at the Pd wire surface with a significant porous structure, which results in an increase of the total surface area of the wire.

\section{Computational simulation}

\subsection{Specifics of the computation}

Since the weakly buoyant flow field is sufficiently simple and well defined, its simulation was performed by using the commercial software package FLUENT to determine the surface heat release rate of a horizontal cylinder maintained at a given temperature. In particular, since the ratio of the wire length to its diameter is large, the flow around the heated wire can be approximated as a 2D natural convection problem. Furthermore, because of axisymmetry, only half of the cylinder was simulated. Figure 1 shows the mesh, which was created by the software GAMBIT, with a maximum cell squish of 0.13 . Quad instead of tri meshes were used for increased accuracy with fewer cells. The area external to the cylinder covering the bulk flow was also resolved with fine meshes because of the importance and complication of the flow around the cylinder. The laminar, viscous flow was solved using pressurebased solver at steady-state conditions. Radiation was not considered because the highest wire temperature was less than $800 \mathrm{~K}$ and the wire was very thin. For pressure-velocity coupling, the SIMPLE (Semi-Implicit Method for Pressure Linked Equations) algorithm was used. The discretization scheme was least squares cell based for the gradient, standard for the pressure, and second order upwind for the momentum, energy and species concentrations $\left(\mathrm{O}_{2}\right.$, $\mathrm{H}_{2} \mathrm{O}, \mathrm{CH}_{4}, \mathrm{CO}$, and $\mathrm{CO}_{2}$ ). Since the velocity in the present natural convection flow is very low, the residue values for continuity and momentum were taken as $10^{-6}$ while that for energy as $10^{-10}$. The boundary conditions are as follows: the cylinder was defined as a wall with the surface reaction mechanism at constant temperature; bottom, top and side surfaces were defined as the pressureinlet type and taken as equal to the ambient temperature; the mole

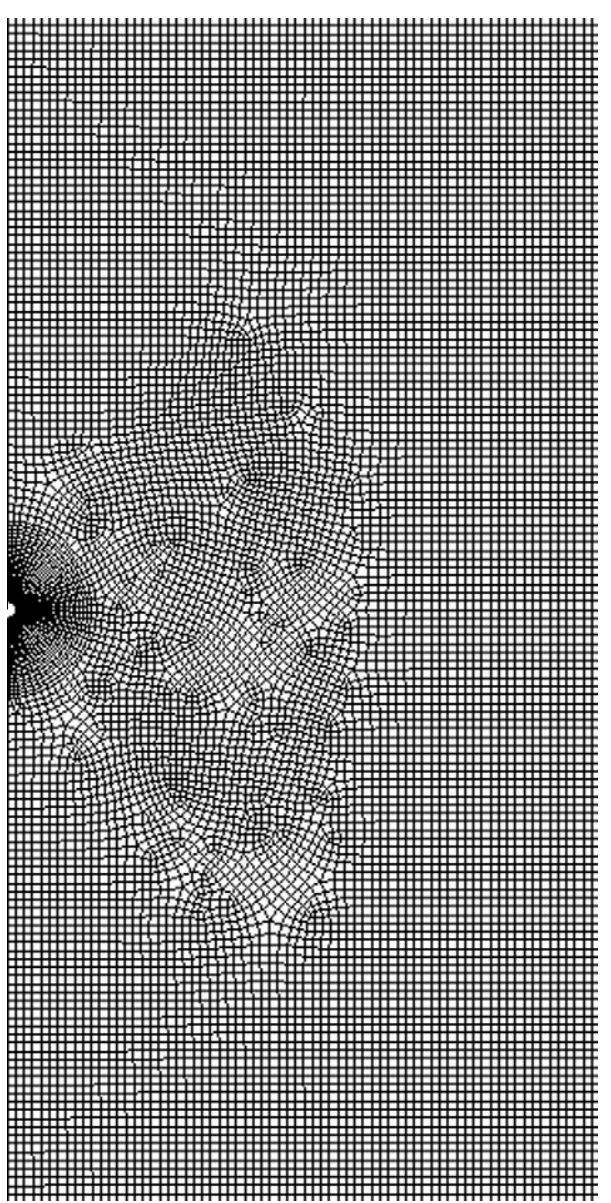

Fig. 1. The grid of the $2 \mathrm{D}$ model of the horizontal cylinder.

fraction of each component in the fuel/air mixture was defined as constant at bottom, top and side surfaces. The height of the mesh domain was twice its width. With increasing width of the mesh, the influence of the mesh domain on the heat flux rate of the cylinder was studied. The result shows that the heat flux rate becomes independent of the domain size when the ratio of the domain's width and cylinder's diameter is larger than 60. Thus the ratio was set at 70 . The surface reaction mechanism was taken from Ref. [1].

\subsection{Validation of the flow model}

The model without considering surface reaction was validated by the relation of Rayleigh number (Ra) and Nusselt number $(\mathrm{Nu})$.

$$
\begin{aligned}
& \mathrm{Ra}=\mathrm{GrPr}=\frac{g \beta}{v \alpha}\left(T_{s}-T_{\infty}\right) D^{3} \\
& \mathrm{Nu}=\frac{\mathrm{hD}}{\kappa}=\frac{q}{A \Delta T} \frac{D}{\kappa}
\end{aligned}
$$

where Gr is the Grashof number, Pr the Prandtl number, $\mathrm{g}$ the gravitational acceleration, $\beta$ the thermal expansion coefficient, $v$ the kinetic viscosity, $\alpha$ the thermal diffusivity, $T_{s}$ the surface temperature, $T_{\infty}$ the environment temperature, $D$ the diameter of the cylinder, $\mathrm{Nu}$ the Nusselt number, $h$ the convective heat transfer coefficient, $\kappa$ the thermal conductivity, $q$ the heat flow, and $A$ the heat transfer surface area, $\Delta T$ difference in temperature of the wire surface and ambient gas. The fluid properties $\operatorname{Pr}, \beta, v, \alpha$ and $\kappa$ were evaluated at the film temperature $\left(T_{f}\right)$, which is defined as $T_{f}=\left(T_{s}+T_{\infty}\right) / 2$. In our case $T_{\infty}$ is the room temperature and $T_{s}$ varies in the range of 


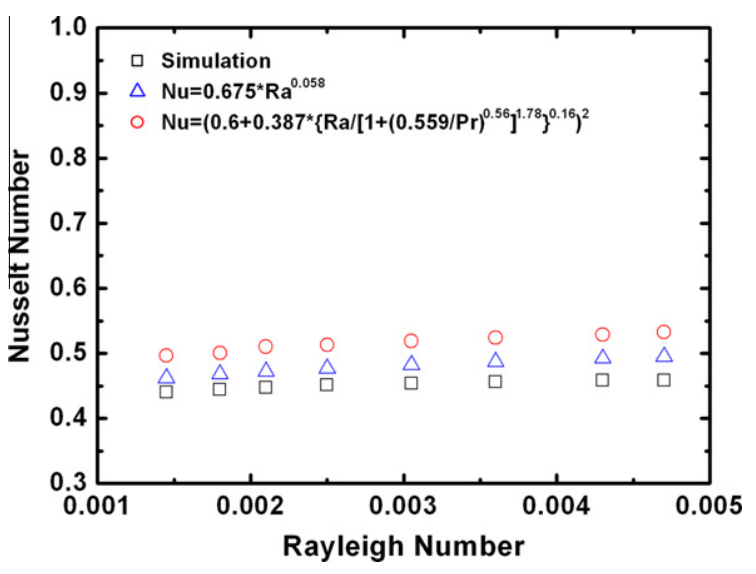

Fig. 2. Dependence of Nusselt Number on Rayleigh Number given by expressions in the literature and determined through the present simulation.

$500-1200 \mathrm{~K}$. Correspondingly, Ra is in the range of $1.5-5 \times 10^{-3}$, implying low Rayleigh number flows. The ratio $q / A$ was calculated, from which $\mathrm{Nu}$ was determined.

As shown in Fig. 2, the black blocks represent the calculated relation between $\mathrm{Ra}$ and $\mathrm{Nu}$, while the red circles and blue triangles denote the relation of $\mathrm{Ra}$ and $\mathrm{Nu}$ calculated by using the following two expressions for free-convection heat transfer [16],

$$
\begin{aligned}
\mathrm{Nu}= & \left(0.60+0.387\left(\frac{\mathrm{Ra}}{\left(1+(0.559 / \mathrm{Pr})^{0.56}\right)^{1.78}}\right)^{0.16}\right)^{2} 10^{-5} \\
& \leqslant \mathrm{Ra} \leqslant 10^{12} \\
\mathrm{Nu} & =0.675 \mathrm{Ra}^{0.058} 10^{-10} \leqslant \mathrm{Ra} \leqslant 10^{-2}
\end{aligned}
$$

It is seen that the values determined by the calculation are very close to those given by the correlations, hence substantiating the adequacy of the computational simulation. It is noted that since the two correlations were obtained by fitting the experimental data in different ranges, they do not overlap in their respective ranges of correlation.

\section{Results and discussion}

\subsection{Heat release rate and effective surface area}

In Fig. 3 we compare the experimental data for the catalytic oxidation of $2 \% \mathrm{CH}_{4}$ with the simulated heat release rate per unit area of the cylinder surface, assuming that it is nonporous. It is seen that the simulated results are substantially lower than the corresponding experimental values, with the former approaching the latter only when the wire temperature approaches $780 \mathrm{~K}$. The comparison therefore implies two aspects of the catalytic ignition not adequately captured by the simulation, namely an under-count of the accessible surface area due to the surface porosity, and some intrinsic weakness of the reaction mechanism. Indeed, our previous work [13] on surface characterization demonstrated that a $1-2 \mu \mathrm{m}$ porous layer was formed at the Pd wire surface, thereby indicating that the surface increase must be considered in the simulation. Furthermore, it is reasonable to expect that the surface area effect dominates at the lower temperature regime, within which the surface reactions are sufficiently slow such that all surfaces are accessible to the reactants through diffusion and reaction is rate controlling.

The increase in the surface area by factors of two and three was considered in Fig. 3. It is seen that these results with augmented surface areas indeed compare closer to the experimental data. Furthermore, since the $2 \times$ results are consistently below the

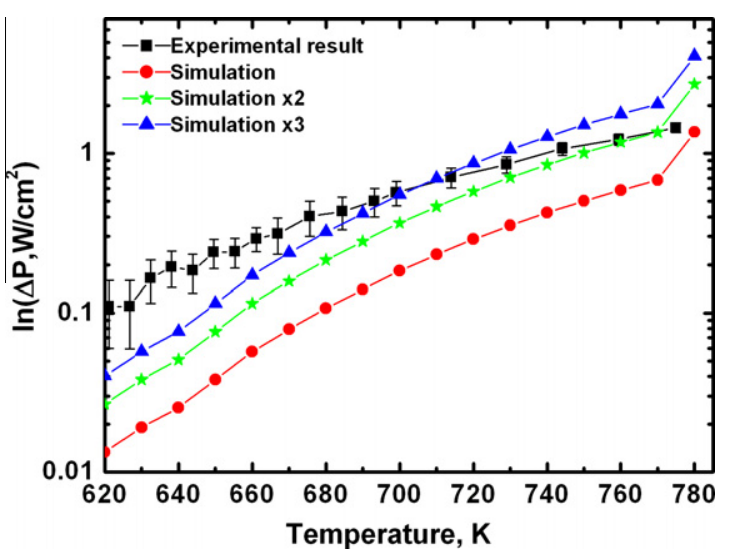

Fig. 3. Comparison of the measured and simulated heat release rate per unit area for the catalytic oxidation of $2 \% \mathrm{CH}_{4}$, allowing for $2 \times$ and $3 \times$ increase in the accessible surface area due to porosity.

experimental data while the $3 \times$ results exceed them at about $700 \mathrm{~K}$, the increase in the surface area should be between $2 \times$ and $3 \times$, which is accordant with the finding of Ref. [17] that an increase in the surface area by a factor of two to three through surface roughing during the chemical transformation Pd metal to Pd oxide.

Figure 4 plots the calculated methane concentration next to the surface at different surface temperatures. It is seen that the concentration maintains a nearly constant value below $700 \mathrm{~K}$, but decreases with increasing temperature afterwards. It is noted that although the system is not solely controlled by diffusion above $700 \mathrm{~K}$, a decreasing trend in the mass fraction of methane at the wire surface highlights the influence of mass diffusion over this range. It is therefore reasonable to consider that reactions occur over all available surfaces including the inner surfaces of the pores below the temperature range of $700 \mathrm{~K}$. With increasing temperature, finite-rate mass diffusion becomes more important, leading to less and less surface area within the pore structure is accessed.

\subsection{Global activation energy and surface mechanism modification}

The global activation energies extracted from the experimental and simulation results were determined and compared. The extraction is based on an overall reaction for complete methane oxidation. A relationship between the surface heat release rate $\Delta P$, and the reaction rate constant $k$ can be expressed as:

$\Delta P / A=k\left[\mathrm{CH}_{4}(\mathrm{~g})\right]^{a} \Delta H$

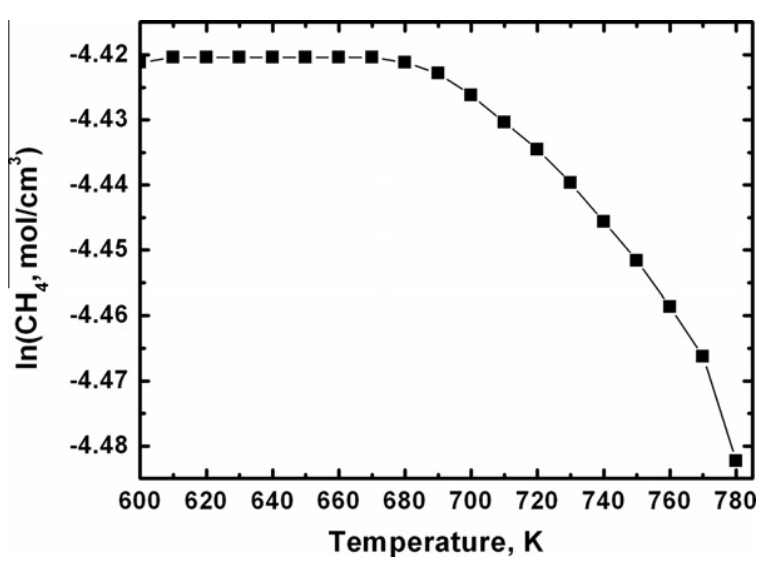

Fig. 4. Simulated mass fraction of methane adjacent to the catalyst with increasing temperature, demonstrating the role of mass diffusion in the transition from low- to high-temperature regimes. 


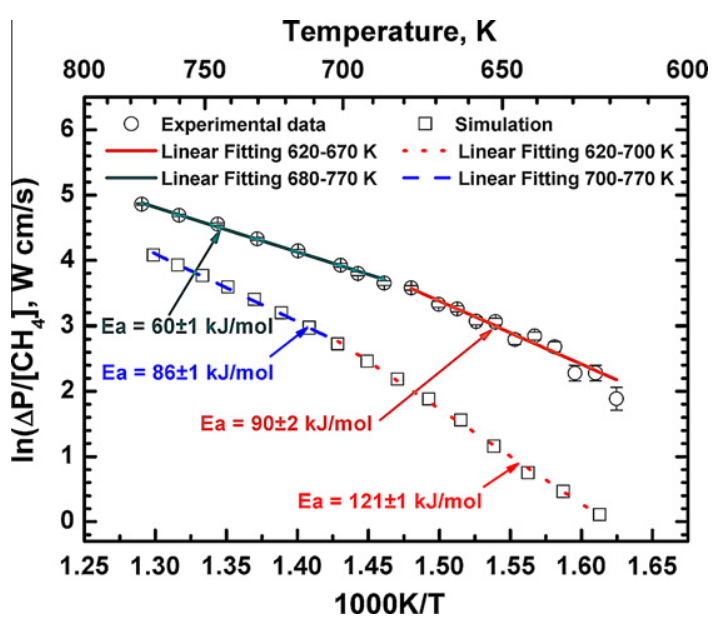

Fig. 5. Global activation energies extracted from the experimental and simulation data.

where $A$ is the surface area, $\left[\mathrm{CH}_{4}(g)\right]$ the ambient methane concentration adjacent to the wire surface, $a$ the reaction order with respect to methane, and $\Delta H$ the enthalpy change of the overall reaction. In writing the above expression it is recognized that oxygen is in excess, and the products water and $\mathrm{CO}_{2}$ are continuously removed by the weakly buoyant flow. The reaction order $a$ has been found to be about 1.0 in $[13,18,19]$. Assuming that $\Delta H$ is not temperature dependent in the present narrow temperature range $(600-780 \mathrm{~K})$, and that the reaction is Arrhenius, we have

$\frac{d \ln \left(\frac{\Delta P}{A\left[\mathrm{CH}_{4}(g)\right]}\right)}{d(1 / T)}=-\frac{E_{a}}{R}+B$.

Figure 5 plots the experimental and simulation results of $\ln \{\Delta P \mid$ $\left.\left(A\left[\mathrm{CH}_{4}(g)\right]\right)\right\}$ versus $1000 / T$ for $2 \%$ methane in the temperature range of $620-770 \mathrm{~K}$, where $A$ is selected as the surface area of the nonporous cylinder. This surface area is proper for simulation study but different from the effective surface area of the experimental study, as discussed in Section 4.1. The reason for not using the effective surface area is that effective surface area is a deduced and inaccurate value, compared with the surface area of the nonporous cylinder. Moreover, it is constant below $700 \mathrm{~K}$, which makes Ea not different from that obtained by using the surface area of the nonporous cylinder. Figure 5 indeed shows a discernible break in the slopes of the experimental results: $90 \pm 2 \mathrm{~kJ} / \mathrm{mol}$ over $620-670 \mathrm{~K}$ and $60 \pm 1 \mathrm{~kJ} / \mathrm{mol}$ over $680-770 \mathrm{~K}$. It is noted that the break in slope occurs around the same temperature of about $700 \mathrm{~K}$ for the simulated results, although the global activation energies are substantially larger than the corresponding experimental values: $121 \pm 1 \mathrm{~kJ} / \mathrm{mol}$ over $620-700 \mathrm{~K}$, and $86 \pm 1 \mathrm{~kJ} / \mathrm{mol}$ over $700-770 \mathrm{~K}$. It is noted that comparison of Ea from experimental and simulated data is worthy only below $700 \mathrm{~K}$, because the effective surface is completely accessible to reactions and as such remains fixed in the low temperature regime below $700 \mathrm{~K}$, not influencing the slope of the plot. Consequently the extracted global activation energy is indeed a kinetic parameter. However, since the effective surface area is expected to change with increasing temperature above $700 \mathrm{~K}$ due to mass diffusion limitation, the activation energy above $700 \mathrm{~K}$ is necessarily affected.

Figure 5 shows the obvious difference between the experimental and simulation results below $700 \mathrm{~K}$, which indicates certain inadequacies of the reaction mechanism. Consequently we shall examine the kinetics in this regime, attempting to improve the reaction mechanism. With the improved mechanism we can then study the behavior in the high-temperature regime.

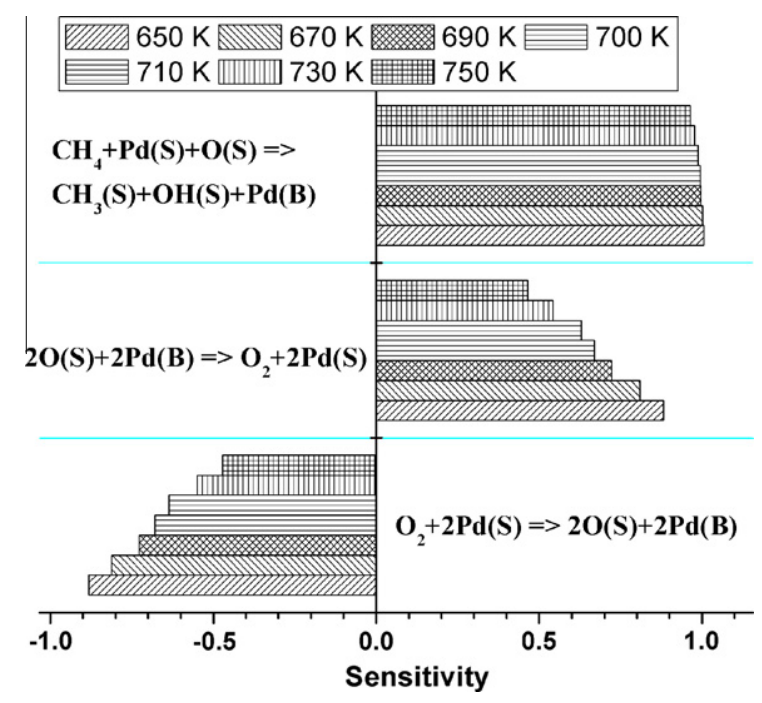

Fig. 6. Sensitivity analysis of the heat release rate for $2 \% \mathrm{CH}_{4}$ at seven different temperatures in the temperature range of $650-750 \mathrm{~K}$. Sensitivity lower than 0.05 not included.

Figure 6 shows the sensitivity analysis of the heat release rate of $2 \% \mathrm{CH}_{4}$ at seven different temperatures over $650-750 \mathrm{~K}$. The sensitivity of the $i$ th surface reaction, $S_{i}$, is defined as

$S_{i}=\frac{\Delta \ln (h r)}{\Delta \ln \left(k_{i}\right)}$,

where $h r$ and $k_{i}$ refer to the simulated heat release rate and the rate coefficient of the $i$ th reaction. This sensitivity is calculated through perturbing the rate coefficient $k_{i}$ by $0.1 \%$.

Three dominant reactions are identified, as also identified in [1]: the oxidative adsorption of methane and desorption of oxygen play a positive role, while the adsorption of oxygen has a negative influence. With increasing temperature the importance of the adsorption and desorption of oxygen reduces, while the oxidative adsorption of methane exerts a large impact. We note in passing that simulations with the mechanism of Sidwell et al. [10] showed a much lower heat release rate for temperatures below $800 \mathrm{~K}$. Compared to the mechanism of Shimizu et al. [1], the mechanism of Sidwell appears to underestimate the importance of the oxidative adsorption of methane and the desorption of oxygen, and overestimate the adsorption of oxygen.

Recognizing that the low-temperature regime, below $700 \mathrm{~K}$, is purely kinetically controlled, we shall optimize the rate coefficients of the three dominant reactions based on our experimental data of a constant effective area. The optimization is based on the response surface methodology [20], which is briefly described here.

Generally, the simulation results are functions of model parameters. For instance, in this work, the simulated heat release rates, $\mathrm{Hr}_{\text {sim }}$, depend on the rate coefficients of the three dominant reactions, say $k_{1}, k_{2}, k_{3}$. Mathematically, $\mathrm{Hr}$ can be represented by a Taylor expansion:

$\mathrm{Hr}_{\text {sim }}=\mathrm{Hr}_{0}+\sum_{i=1}^{3} a_{i} \Delta k_{i}+\sum_{i=1}^{3} \sum_{j \geqslant i}^{3} b_{i j} \Delta k_{i} \Delta k_{j}+\cdots$

where $a_{i}$ and $b_{i j}$ characterize the sensitivity of $\mathrm{Hr}_{\text {sim }}$ to perturbations of rate coefficients and their cross terms. When the rate coefficients vary in a constrained local range of the parameter space, $a_{i}$ and $b_{i j}$ are almost constant. Therefore, $a_{i}$ and $b_{i j}$ can be derived from a few tentative computations with slightly varied rate coefficients. Subsequently, corresponding to the experimentally measured heat release rate $\mathrm{Hr}_{\text {exp }}$, the optimized rate coefficients can be inversely 


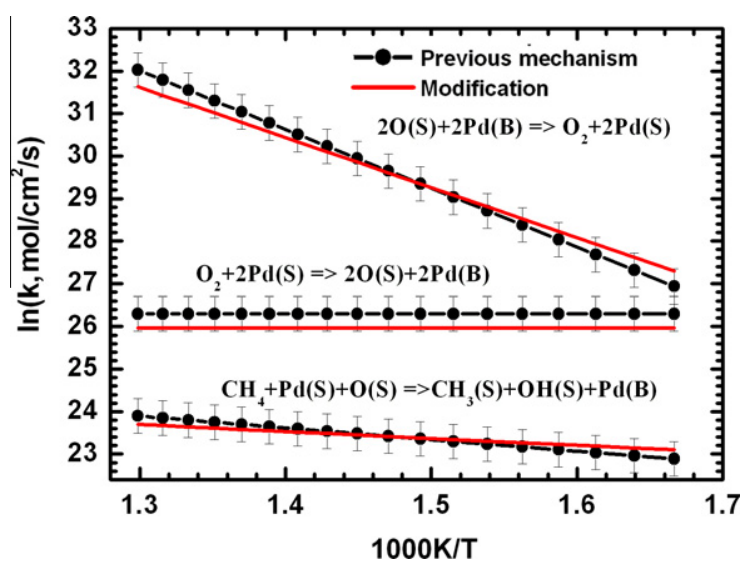

Fig. 7. Optimized rate coefficients of the three dominant reactions in the modified model.

Table 1

Optimized reaction rate coefficients of the three dominant reactions (values from the original model indicated in parenthesis).

\begin{tabular}{llll}
\hline Reactions & $A$ & $\beta$ & $\begin{array}{l}\mathrm{Ea} \\
(\mathrm{kJ} / \mathrm{mol})\end{array}$ \\
\hline $\mathrm{O}_{2}+2 \mathrm{Pd}(\mathrm{s}) \rightarrow 2 \mathrm{O}(\mathrm{s})+2 \mathrm{Pd}(\mathrm{B})$ & $0.6(0.8)^{\mathrm{a}}$ & $-0.5(-0.5)$ & $0.0(0.0)$ \\
$2 \mathrm{O}(\mathrm{s})+2 \mathrm{Pd}(\mathrm{B}) \rightarrow \mathrm{O}_{2}+2 \mathrm{Pd}(\mathrm{s})$ & $2.60 \mathrm{E}+20$ & $0.0(0.0)$ & $230.0-132$ \\
& $(5.13 \mathrm{E}+21)^{\mathrm{b}}$ & & $(115) \theta_{\mathrm{o}}$ \\
& $7.7 \mathrm{E}-3$ & $0.0(0.0)$ & $10.6(20.0)$ \\
$\mathrm{CH}_{4}+\mathrm{Pd}(\mathrm{s})+\mathrm{O}(\mathrm{s}) \rightarrow \mathrm{CH}_{3}(\mathrm{~s})$ & $(4.2 \mathrm{E}-2)^{\mathrm{a}}$ & & \\
$+\mathrm{OH}(\mathrm{s})+\mathrm{Pd}(\mathrm{B})$ & & &
\end{tabular}

${ }^{a}$ Sticking coefficient.

b Unit for $A$ factor is $\mathrm{cm}^{2} \mathrm{~mol}^{-1} \mathrm{~s}^{-1}$.

\section{Temperature, $\mathrm{K}$}

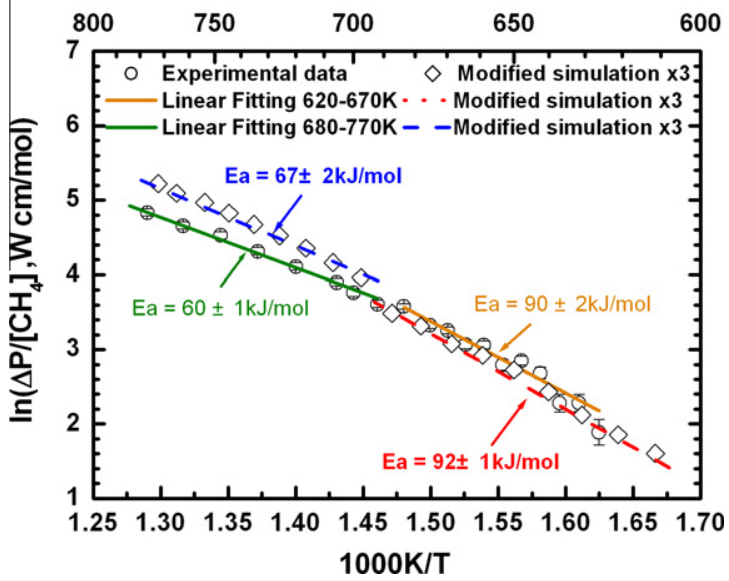

Fig. 8. Comparison of global activation energies deduced from the experimental data and the simulation with the optimized mechanism.

solved. In our case, for a temperature range over $600-700 \mathrm{~K}, \mathrm{Hr}_{\text {sim }}$ is expressed by an expansion of $\Delta k_{i}$ up to second-order, which is sufficient for most combustion problems. The rate coefficients are adjusted within $\pm 50 \%$ of the original model, which is less than the common factor-of-two uncertainty in model development. The optimized rate coefficients are given in Fig. 7 and Table 1: the difference between the modified and the original rate coefficients is less typically than $25 \%$ at various temperatures.

Figure 8 shows the global activation energies deduced by the simulation results with the modified model. It is seen that, below $700 \mathrm{~K}$, the predicted global activation energy of $92 \pm 1 \mathrm{~kJ} / \mathrm{mol}$ is fairly close to that from experimental measurement of $90 \pm 2 \mathrm{~kJ} /$ mol. In the high-temperature range, the predicted value is

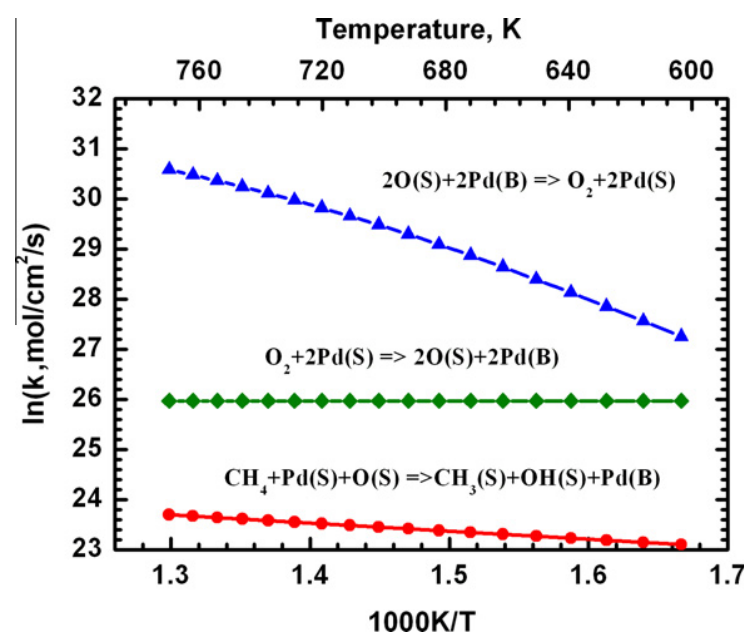

Fig. 9. Arrhenius plots for the three key catalytic reactions.

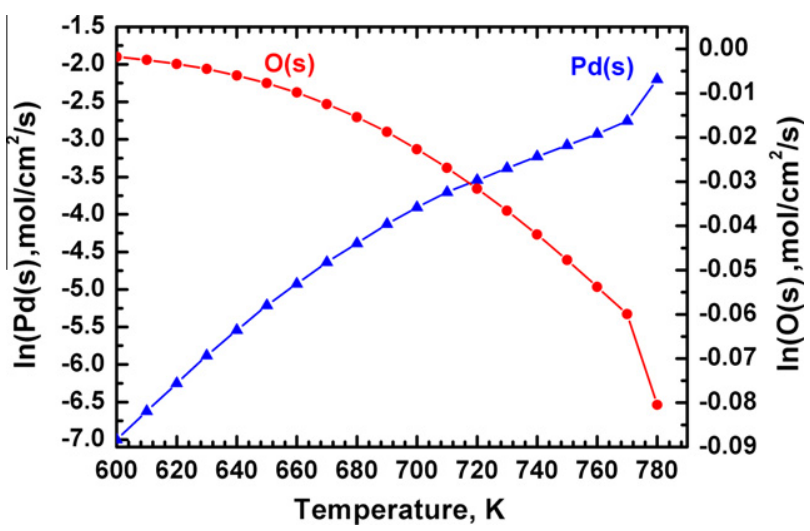

Fig. 10. Prediction fractional surface coverage of $\mathrm{Pd}(\mathrm{s})$ and $\mathrm{O}(\mathrm{s})$ on the catalyst surface with increasing temperature.

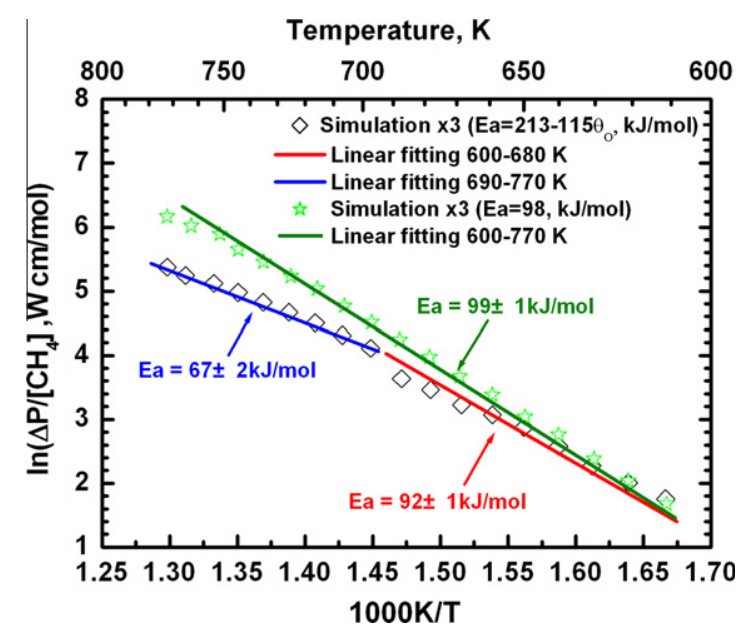

Fig. 11. Comparison of experimental activation energies and simulation with a constant oxygen desorption energy of $98 \mathrm{~kJ} / \mathrm{mol}$

$67 \pm 2 \mathrm{~kJ} / \mathrm{mol}$. It is noted that the variation of effective surface area was not considered in the prediction in the high temperature range. However, it is still interesting that there is a huge difference between two predicted activation energies in the two temperature range. 


\subsection{Kinetic cause in transition in global activation energy}

With the improved kinetic mechanism, we now further study its kinetic implications. Figure 9 plots the rate constants of the three dominant reactions in the Arrhenius form over the temperature range $600-780 \mathrm{~K}$, showing linear variation for the rate constants of oxygen (green diamonds) ${ }^{1}$ and methane (red circles) adsorption, while that of oxygen desorption (blue triangles) varies nonlinearly with increasing temperatures above $700 \mathrm{~K}$. This therefore implies constant activation energy for oxidation and methane adsorption, and a varying one for oxygen desorption above $700 \mathrm{~K}$. The transition comes from the oxygen coverage effect on the activation energy of oxygen desorption, $\mathrm{Ea}(\mathrm{kJ} / \mathrm{mol})=230-132 \theta_{\mathrm{O}}$ (also shown in Table 1 ). Specifically, Fig. 10 shows that the coverage of $\mathrm{O}(\mathrm{s})$ is close to unity below $700 \mathrm{~K}$, but decreases substantially above $700 \mathrm{~K}$ and as such affects the activation energy of oxygen desorption, which in turn affects the global activation energy, as observed herein both experimentally and computationally. To further substantiate this hypothesis, simulations with constant oxygen desorption energy of $98 \mathrm{~kJ}$ were conducted, yielding a constant global activation energy of $99 \pm 1 \mathrm{~kJ} / \mathrm{mol}$, with no apparent break in the slope, as shown in Fig. 11.

\section{Concluding remarks}

In the present investigation we have further demonstrated the usefulness of the microcalorimetry technique in the study of heterogeneous catalytic reactions. Because of the simple and welldefined nature of the design, it can be experimentally set up and computationally simulated expediently and with good accuracy. While the observables are limited to the global properties of the heat release rate as a function of temperature, with the simultaneous determination of the ignition temperature, the controlling reactions can be assessed through simulation with detailed chemistry. Indeed, the present approach is similar to that of measuring the laminar flame speed of a reactive mixture and extracting useful chemical information through simulation with some given reaction mechanism.

For the present problem of the catalytic oxidation of methane/ air over a Pd wire with a porous 1-2 $\mu \mathrm{m}$ PdO surface layer, the experimental and simulation results demonstrate that the catalytic oxidation is characterized by a low-temperature, reaction-controlled regime in which the internal pore surface is totally accessible to the reactions, and a high-temperature regime in which finite-rate pore diffusion also affects the overall heat release rate. Furthermore, the heat release rate is found to be primarily affected by the oxidative adsorption of methane together with the adsorption and desorption of oxygen, and the temperature-dependent $\mathrm{O}(\mathrm{s})$ surface coverage strongly affects the transition from the low- to the high-temperature regimes.
Quantitatively useful information acquired from the present study include an optimized catalytic reaction mechanism with the attendant revision of the key reaction rate constants from literature values; the determination of the transition temperature of $700 \mathrm{~K}$ from the kinetically controlled low-temperature regime to the diffusion-affected high-temperature regime, with the associated global activation energies of $92 \pm 1 \mathrm{~kJ} / \mathrm{mol}$ for the catalytic ignition; and the demonstration that the effective surface area allowing for the porous structure is about 2-3 times larger than the nonporous surface, in agreement with the independently measured value in the literature. Our study on the improved kinetic mechanism further demonstrates the significant effect of surface coverage on the activation energy.

\section{Acknowledgments}

The work at Princeton University was supported by the Air Force Office of Scientific Research. Taichang Zhang was a visiting student from the University of Science and Technology of China to Princeton University during the early phase of this investigation; the work was completed after his return to China. The authors would like to thank Professor Hai Wang of the University of Southern California for helpful discussions.

\section{References}

[1] T. Shimizu, A.D. Abid, G. Poskrebyshev, H. Wang, J. Nabity, J. Engel, J. Yu, D. Wickham, B. Van Devener, S.L. Anderson, S. Williams, Combust. Flame 157 (2010) 421-435.

[2] D. Ciuparu, M.R. Lyubovsky, E. Altman, L.D. Pfefferle, A. Datye, Catal. Rev. - Sci Eng. 44 (2002) 593-649.

[3] J.N. Carstens, S.C. Su, A.T. Bell, J. Catal. 176 (1998) 136-142.

[4] M. Valden, J. Pere, M. Hirsimaki, S. Suhonen, M. Pessa, Surf. Sci. 377 (1997) 605-609.

[5] E. Broclawik, R. Yamauchi, A. Endou, M. Kubo, A. Miyamoto, J. Chem. Phys. 104 (1996) 4098-4104

[6] R. Burch, D.J. Crittle, M.J. Hayes, Catal. Today 47 (1999) 229-234.

[7] J.C. van Giezen, F.R. van den Berg, J.L. Kleinen, A.J. van Dillen, J.W. Geus, Catal. Today 47 (1999) 287-293.

[8] D.N. Gao, S. Wang, C.X. Zhang, Z.S. Yuan, S.D. Wang, Chin. J. Catal. 29 (2008) 1221-1225.

[9] J. Cortes, E. Valencia, P. Araya, J. Phys. Chem. C 114 (2010) 11441-11447.

[10] R.W. Sidwell, H.Y. Zhu, R.J. Kee, D.T. Wickham, C. Schell, G.S. Jackson, Proc. Combust. Inst. 29 (2003) 1013-1020.

[11] R.W. Sidwell, H.Y. Zhu, R.J. Kee, D.T. Wickham, Combust. Flame 134 (2003) 5566.

[12] M.M. Wolf, H.Y. Zhu, W.H. Green, G.S. Jackson, Appl. Catal. A-Gen 244 (2003) $323-340$.

[13] T.C. Zhang, D.L. Zhu, N. Yao, F. Qi, C.K. Law, Proc. Combust. Inst. 33 (2011) 1819-1825.

[14] L. Hiam, H. Wise, S. Chaikin, J. Catal. 10 (1968) 272-276.

[15] P. Cho, C.K. Law, Combust. Flame 66 (1986) 159-170.

[16] A. Bejan, A.D. Kraus, Heat transfer handbook, Wiley, New York, USA, 2003.

[17] J.Y. Han, G.H. Zhu, D.Y. Zemlyanov, F.H. Ribeiro, J. Catal. 225 (2004) 7-15.

[18] C.F. Cullis, B.M. Willatt, J. Catal. 83 (1983) 267-285.

[19] F.H. Ribeiro, M. Chow, R.A. Dalla Betta, J. Catal. 146 (1994) 537-544.

[20] D.A. Sheen, H. Wang, Combust. Flame 158 (2011) 2358-2374. 